\title{
Hydraulic Fluid Properties and their Impact on Energy Efficiency
}

\author{
Karl-Erik Rydberg \\ Division of Fluid and Mechatronic Systems, Department of Management and Engineering \\ Linköping University, Linköping, Sweden \\ karl-erik.rydberg@liu.se
}

\begin{abstract}
To meet the increased requirements on higher efficiency and better functionality of hydraulic systems, new components and system concepts have been developed over the years. However, the most important component in a hydraulic system, which has a major impact on system efficiency and wear are the fluid itself. The last decades, major attention on hydraulic fluid development, have been set upon environmental adaption. Today, energy efficiency has been a hot topic and a number of energy efficient fluids have been launched on the market. Besides energy efficiency, environmental adaption of fluids, are extremely important in mobile applications.

In this paper the focus is set on hydraulic fluid properties and its impact on system efficiency. Environmental adapted fluids will also be discussed. Synthetic saturated esters are analysed and compared with mineral oils. The main question that will be tried to answer is - "How to select the best fluid for a specific application?".
\end{abstract}

Keywords: Hydraulic fluids, Synthetic esters, Fluid properties, Viscosity, Energy efficiency.

\section{Introduction}

The pressure media is the most important component in the hydraulic system because it takes care of the energy transfer in the whole system from pump to cylinder / motor. Additionally the hydraulic fluid has to provide lubrication and cooling. Especially in mobile applications it is of great importance to minimize the negative environmental influence from hydraulics.

\subsection{Environmental adapted fluids}

In order to make hydraulic fluids environmentally adapted a number of synthetic fluids have been developed. One of the most promising fluids is saturated synthetic esters. This type of fluid has many interesting properties to explore. Synthetic saturated esters have been on the market for more than 10 years, but the evaluation of their properties is still limited. However, the results so far about the lubrication properties, viscosity index, and stability etc are very positive, see Fig. 1. There is no doubt that the synthetic esters can be considered as a future product with a high development potential.
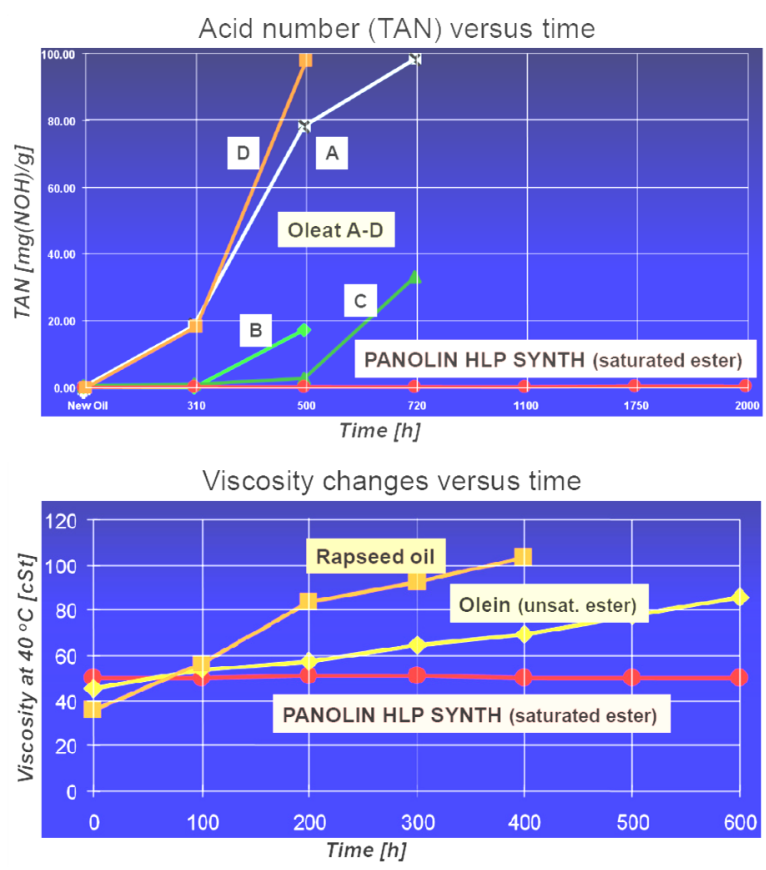

Fig. 1: Acid number and viscosity stability of saturated ester, Panolin HLP Synth, [2]. 
From Fig. 1 it can be seen that the saturated ester (Panolin HLP Synthetic) has extremely stable acid number (TAN) and viscosity compared to unsaturated esters. In comparison with mineral oil the saturated ester also shows impressive shear stability over the time.

\subsection{Energy efficient fluids}

In order to maximize energy efficiencies in hydraulic systems, mechanical and volumetric losses must be balanced so the sum of these losses is minimized. Since the mechanical losses in sealing gaps are proportional to the fluid viscosity and volumetric losses inverse proportional to viscosity, it is clear that an optimal viscosity exists.

Fluid viscosity is not the only parameter that improves energy efficiency. Also, friction coefficient provided by the fluid in sealing gaps as well as the viscosity index (VI) of the fluid is very important parameters. Looking at the development of engine lubricants for cars it can be noted that today's fluids are low viscosity oils with friction modifiers and VI improvers. It has been proven that this kind of fluids have a significant impact on fuel consumption.

The fluid properties impact on hydraulic system energy efficiency has been clearly validated in a NSF-sponsored project at Center for Compact and Efficient Fluid Power (CCEFP) in US. An excavator has been selected as a primary vehicle for a test bed, [3]. As a member of this project, Evonik RohMax has developed a new multi-grade fluid, optimized with high-tech polymer additives and friction modifiers. Field tests, performed by Evonik RohMax, on excavators show efficiency gains between 18 and $26 \%$, depending on work cycle, [4]. The importance of high viscosity index (VI>160) and good shear stability has also been stated.

\subsection{Fluid viscosity grade and viscosity index}

Selection of an energy efficient fluid for a specific hydraulic application is highly associated with a proper viscosity grade and the viscosity index (VI) of the fluid.

The standard viscosity grade, ISO VG gives the fluid viscosity in $\mathrm{cSt}$ at the temperature $40{ }^{\circ} \mathrm{C}$. In mobile applications the most commonly used viscosity grade is VG 46. However, if the hydraulic system contains high speed pumps and motors as well as long lines a lower
VG-value will boost the efficiency. Using fluids with low VG-value (VG 32 or VG20) in mobile machines it is very important to take the viscosity index into account. The lower VG-value the higher VI-value is required.

In mobile applications where the fluid temperature variations can be very high the viscosity index (VI) has a high impact on energy efficiency. RohMax in US, a leading manufacturer of hydraulic fluid additives, has investigated the impact of VI on hydraulic pump efficiency in mobile application, [5]. They have developed a fluid with very high shear stability and tested the fluid in Eaton-Vickers vane pumps. The efficiency improvements versus fluid temperature at different VI-values are shown in Fig. 2. The 0-level stays for $\mathrm{VI}=100$.

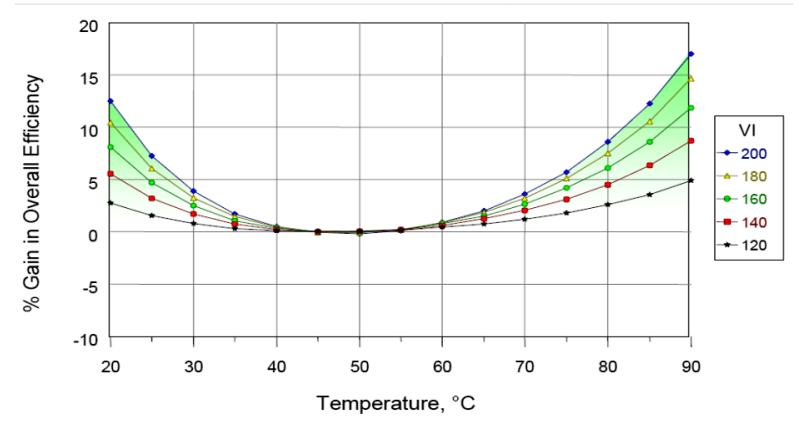

Fig. 2: Gain in pump efficiency versus fluid temperature with VI as parameter, [5].

The above figure shows that VI $>160$ is needed if the fluid temperature rang is bigger than $50{ }^{\circ} \mathrm{C}$. It is also worth to be mentioned that these figures belongs to one specific pump. In practice, the required VI-value depends upon the type of pump, the pump speed range and the system pressure range.

\section{Viscosity dependent losses in hydraulic pipes}

Hydraulic systems are commonly included long pipe. The pressure losses in a straight pipe are theoretically calculated as follows:

Laminar flow: $\quad \Delta p_{\text {lam }}=\frac{32 \cdot L \cdot \eta}{d^{2}} \cdot v_{m}$

Turbulent flow: $\Delta p_{\text {turb }}=\frac{0,068 \cdot L}{d^{1.25}} \cdot \rho^{0.75} \cdot \eta^{0.25} \cdot v_{m}^{1.75}$

where $\boldsymbol{d}=$ pipe diameter [m], $\boldsymbol{L}=$ pipe length $[\mathrm{m}], \boldsymbol{\eta}=$ dynamic viscosity $\left[\mathrm{Ns} / \mathrm{m}^{2}\right], \boldsymbol{\rho}=$ fluid density $\left[\mathrm{kg} / \mathrm{m}^{3}\right], \boldsymbol{v}_{\boldsymbol{m}}$ $=$ average flow velocity $[\mathrm{m} / \mathrm{s}]$.

Calculated pressure drop across a $15 \mathrm{~m}$ long pipe with 
two different viscosities of the fluid (VG 46), are illustrated in Fig. 3.

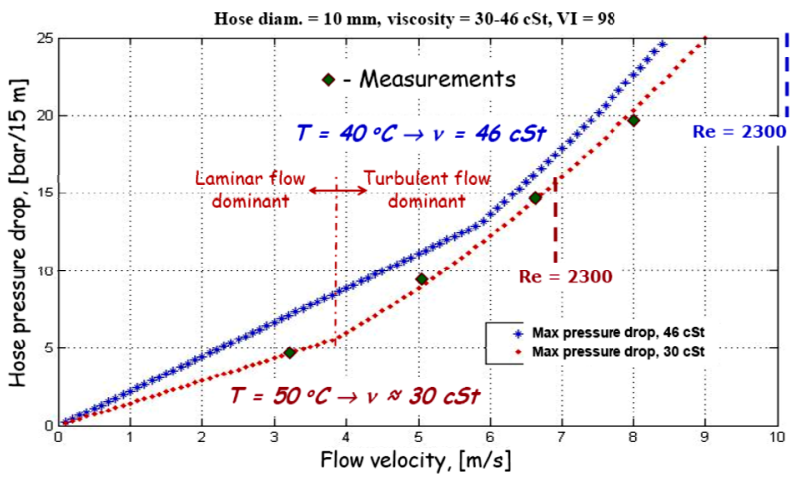

Fig. 3: Pressure drop in a $15 \mathrm{~m}$ long pipe $(\phi=10 \mathrm{~mm})$ versus flow velocity for the viscosity 30 and $46 \mathrm{cSt}$.

Comparison with measurements in Fig. 3, shows that the real pressure drop follows the theoretical values if this is calculated as,

$$
\Delta p=\max \left\{\Delta p_{\text {lam }}, \Delta p_{\text {turb }}\right\}
$$

From the figure it can be observed that the turbulent flow starts to increase the pressure drop at a much lower Re-number than the critical value (2300). In Fig 4, $\Delta p_{\text {turb }}$ is bigger than $\Delta p_{\text {lam }}$ for all $\operatorname{Re}>0,56 * 2300$. In practice, the transition from laminar to turbulent stage is very smooth and not as an immediate switch at $\mathrm{Re}=2300$.

\subsection{Pressure drop measurements}

Pressure drop measurements have been performed at the division of Fluid and Mechatronic Systems in Linkoping, Sweden. The principle layout of the used test-stand is illustrated in Fig. 4.

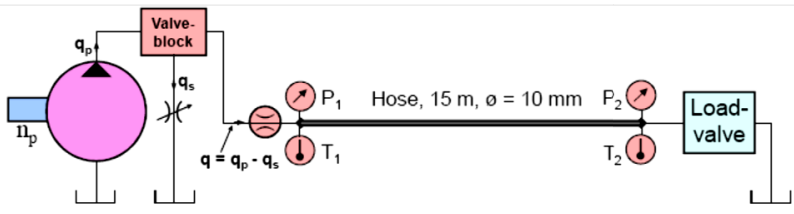

Fig. 4: Test-stand for measurements of pressure drop in pipes.

The test object is $15 \mathrm{~m}$ long hose with an inner diameter of $10 \mathrm{~mm}$. The fluids that have been tested are one mineral oil (VG 46), four different synthetic saturated esters (three VG 32 fluids and one VG 12) and one low viscosity fluid an eco-diesel (VG 3). Measured hose pressure drop versus fluid velocity for these six fluids are shown in Fig. 5. More details about the pressure drop tests and fluid examinations are presented in ref. [6].
The test results demonstrated in Fig. 5, are all following the theoretical calculations according to eq. (3).

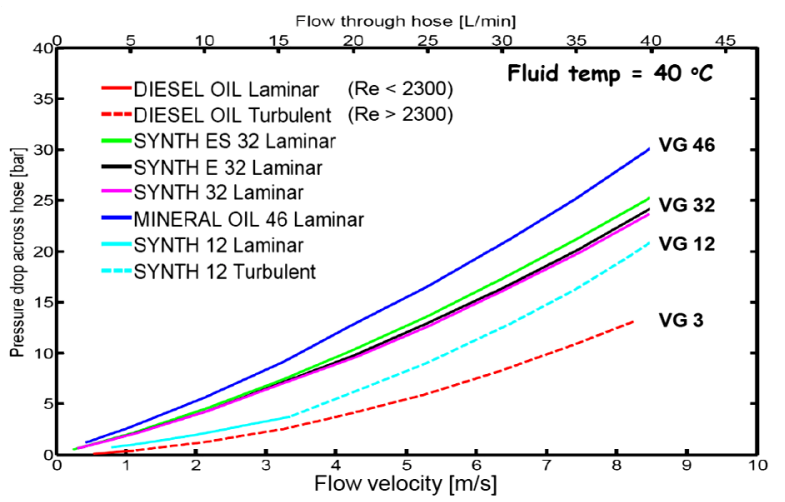

Fig. 5: Measured pressure drop in a $15 \mathrm{~m}$ long hose $(\phi=10 \mathrm{~mm})$ versus flow velocity for 6 different fluids.

\section{Energy efficiency of pumps and motors}

All hydraulic systems are containing at least one pump. The pump is a primary system unit that has to transform mechanical energy into hydraulic energy. Therefore, the pump efficiency has a significant impact on the overall system efficiency. The pump efficiency is related to the dominant losses, which are volumetric (leakage) and mechanical (friction) losses. These losses are mainly affected by the fluid viscosity. In order to analyses the viscosity impact on pump efficiency a model can be used.

Based on a semi-empirical efficiency model for axial piston machines, [1], Fig. 6 shows simulated pump efficiencies as a function of fluid viscosity.
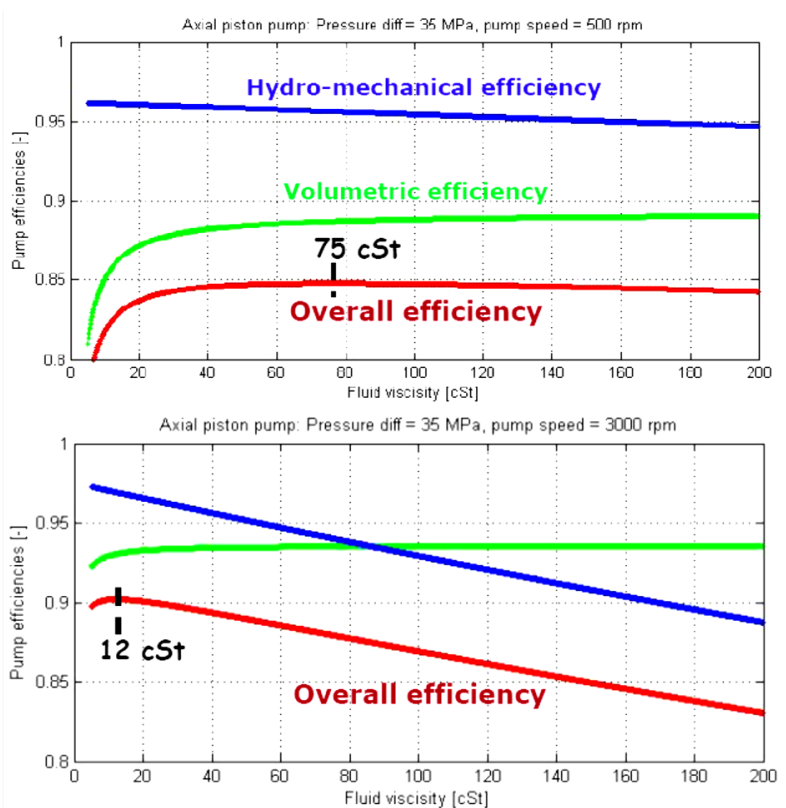

Fig. 6: Efficiencies versus fluid viscosity for an in-line axial piston pump at two speeds, 500 and $3000 \mathrm{rpm}$. 
Fig. 6 shows that the viscosity, which maximize the pump overall efficiency is related to operation conditions, pressure and speed.

From the efficiency model the dynamic viscosity $(\eta)$ that gives max efficiency can be expressed as,

$$
\eta_{o p t}=\frac{p_{p}}{n_{p}} \cdot \sqrt{\frac{C_{v}}{k_{v}}}
$$

where $\boldsymbol{C}_{\boldsymbol{v}}$ and $\boldsymbol{k}_{\boldsymbol{v}}$ are machine constants, $\boldsymbol{p}_{\boldsymbol{p}}=$ pump pressure and $\boldsymbol{n}_{\boldsymbol{p}}=$ pump speed.

Equation (4) is applicable on both pump and motors, but it has to be observed that the machine constants can varies in a wide range, depending on the type of machines.

\subsection{Hydrostatic transmission efficiency}

Hydrostatic transmissions are widely used in drive train for working machines. In a wheel loader or forwarder (forest machine) the drive transmission stays for more than $50 \%$ of the energy consumption. Therefore, it is highly important to make an analyses of how the fluid properties will affect the transmission overall efficiency.

The transmission investigated in this study is schematically depicted in Fig. 7.

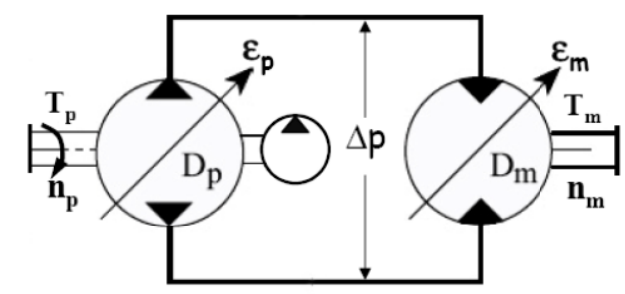

Fig. 7: Schematic drawing of the test transmission.

The transmission concept is traditional, with variable displacement axial piston units as pump (In-line) and motor (Bent-axis). The main pump is supported with a fixed displacement boost pump.

For theoretical examination of transmission efficiency the pump and motor loss models, developed for axial piston units, reference [1], is used.

The variable pump, volumetric and hydro-mechanical efficiencies are expressed as.

$$
\eta_{v p}=1-a_{0 p}-\left(\frac{a_{1 p}}{\varepsilon_{p}}+a_{2}\right) \frac{\Delta p}{\beta_{e}}-a_{3 p} \frac{\Delta p}{2 \pi \eta \varepsilon_{p} n_{p}}-a_{4 p} \frac{\Delta p^{2}}{\varepsilon_{p} n_{p}}
$$

$$
\eta_{l m p}=\frac{1}{1+\frac{b_{0 p}}{\varepsilon_{p}}+b_{1 p}+\left(\frac{b_{2 p}}{\varepsilon_{p}}+b_{3 p}\right) \frac{p_{L}}{\Delta p}+b_{4 p} \frac{p_{H}+\delta_{p} p_{L}}{\left[1+\left(\frac{n_{p}}{n_{0 p}}\right)^{\gamma_{p}}\right] \varepsilon_{p} \Delta p}+b_{s p} \frac{2 \pi \eta n_{p}}{\varepsilon_{p} \Delta p}+b_{6 p} \frac{\varepsilon_{p}^{2} n_{p}^{2}}{\Delta p}}
$$

The efficiencies of the variable motor are,

$$
\begin{gathered}
\eta_{v m}=\frac{1}{1+a_{0 m}+\left(\frac{a_{1 m}}{\varepsilon_{m}}\right) \frac{\Delta p}{\beta_{e}}+a_{2 m} \frac{\Delta p}{2 \pi \eta \varepsilon_{m} n_{m}}+a_{3 m} \frac{\Delta p^{2}}{\varepsilon_{m} n_{m}}} \\
\eta_{l m m}=1-\frac{b_{0 m}}{\varepsilon_{m}}+b_{1 m}+\left(\frac{b_{2 m}}{\varepsilon_{m}}+b_{3 m}\right) \frac{p_{L}}{\Delta p}+b_{4 m} \frac{p_{H}+\delta_{p} p_{L}}{\left[1+\left(\frac{n_{m}}{n_{0 m}}\right)^{\gamma_{m}}\right] \varepsilon_{m} \Delta p}+b_{s m} \frac{2 \pi \eta n_{m}}{\varepsilon_{m} \Delta p}+b_{6 m} \frac{\varepsilon_{m}^{2} n_{m}^{2}}{\Delta p}
\end{gathered}
$$

In equation (5) - (8), $\boldsymbol{a}$ and $\boldsymbol{b}$ are machine dependent loss coefficients, $\eta=$ fluid dynamic viscosity, $\Delta p=$ transmission pressure difference, $\boldsymbol{n}=$ shaft speed and $\boldsymbol{\varepsilon}=$ displacement setting.

The overall transmission efficiency, with exclusion of the boost pump, is calculated as,

$$
\eta_{t, o}=\frac{T_{m} \cdot n_{m}}{T_{p} \cdot n_{p}}=\eta_{v p} \cdot \eta_{h m p} \cdot \eta_{v m} \cdot \eta_{h m m}
$$

Including the boost pump power, the overall efficiency is expressed as,

$$
\eta_{t, b p, o}=\frac{T_{m} \cdot n_{m}}{\left(T_{p}+T_{b p}\right) \cdot n_{p}}
$$

where $\boldsymbol{T}=$ shaft torque.

\subsection{Theoretical analysis of transmission efficiency}

In the analysed transmission (see Fig 7), the main pump and motor have max displacement of $D_{p}=110$ $\mathrm{cm}^{3} / \mathrm{rev}$ and $\mathrm{D}_{\mathrm{m}}=150 \mathrm{~cm}^{3} / \mathrm{rev}$, respectively. The boost pump displacement is $\mathrm{D}_{\mathrm{bp}}=0,2 \cdot \mathrm{D}_{\mathrm{p}}$. Simulated efficiency characteristic of the sequence controlled transmission, without boost pump, is presented in Fig. 8 .

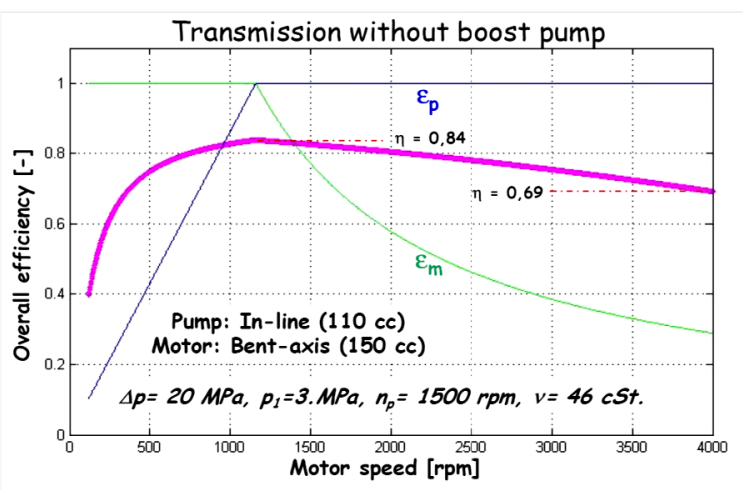

Fig. 8: Simulated transmission overall efficiency versus motor speed, without boost pump, $v=46 \mathrm{cSt}$.

The transmission efficiency characteristic in Fig. 8 is calculated for operation conditions of constant pump 
speed, $\mathrm{n}_{\mathrm{p}}=1500 \mathrm{rpm}$, constant pressure difference, $\Delta \mathrm{p}=$ $20 \mathrm{MPa}$, constant low pressure (boost pressure), $\mathrm{p}_{1}=3$ $\mathrm{MPa}$ and a fluid viscosity of $46 \mathrm{cSt}$. Max efficiency $(84 \%)$ is reached, as expected, when the pump and motor have max displacement stroking $\left(\varepsilon_{\mathrm{p}}=\varepsilon_{\mathrm{m}}=1.0\right)$. When the motor speed is increased from $1000 \mathrm{rpm}$ to $4000 \mathrm{rpm}$ the efficiency goes down, mainly because of viscosity and speed dependent friction and pressure losses. At the motor speed, $4000 \mathrm{rpm}$ the transmission efficiency is reduced to $69 \%$.

If the boost pump is included in the efficiency calculations (Eq. 10), the efficiency graph became as in Fig. 9. At constant pump speed the boost pump power is constant, around $3 \%$ of the main pump power, when $\varepsilon_{\mathrm{p}}=1.0$ and $\Delta \mathrm{p}=20 \mathrm{MPa}$. Therefore, the boost pump will reduce the transmission overall efficiency approximately $3 \%$ (when $\varepsilon_{\mathrm{p}}=1.0$ ), compared to the case in Fig. 8.

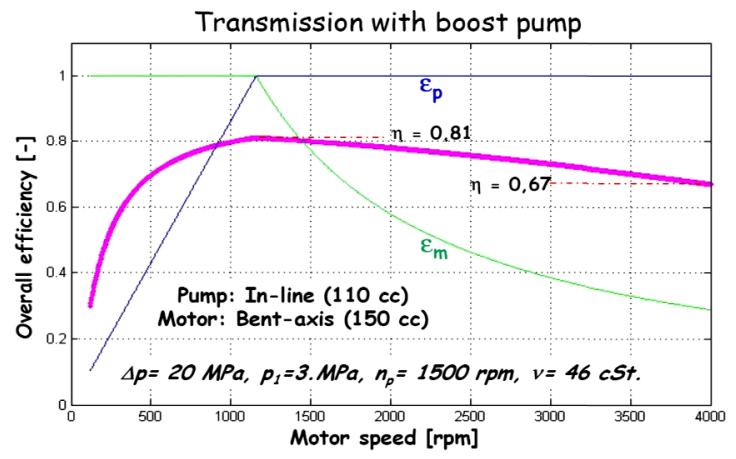

Fig. 9: Simulated transmission overall efficiency versus motor speed, with boost pump, $v=46 \mathrm{cSt}$.

According to equation (4), the fluid viscosity that maximizes the efficiency of a hydraulic machine, depends upon pressure and speed. By using a low viscosity fluid (VG 12) in the transmission, it can be expected that its efficiency will be mostly gained at high motor speed. This is confirmed by the characteristics shown in Fig. 10.

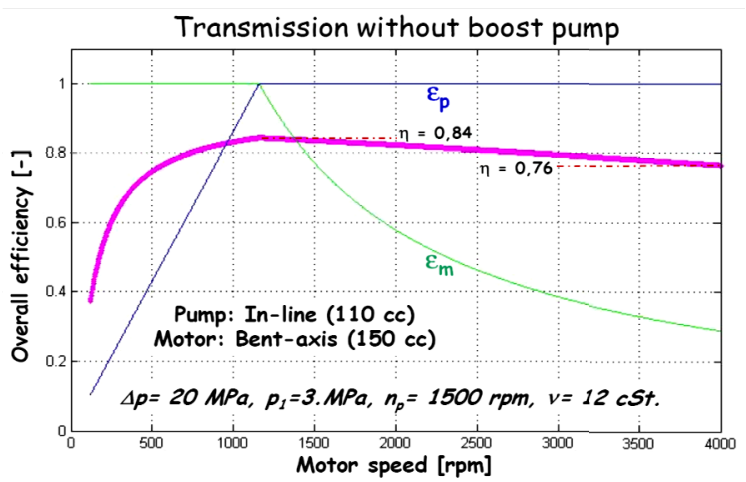

Fig. 10: Simulated transmission overall efficiency versus motor speed, without boost pump, $v=12 \mathrm{cSt}$.
Energy efficient hydraulic fluids, is the commonly used name for fluids containing specific friction modifiers. For mobile applications these fluids also have high viscosity index $(\mathrm{VI}>150)$ and high shear stability, [7].

Focusing on the friction modifiers impact on transmission efficiency, this can be evaluated by reduction of the friction coefficient values in the efficiency model (eq. 5-8). In Fig. 11, the transmission efficiency characteristics (boost pump included) are shown for $\Delta \mathrm{p}=40 \mathrm{MPa}$, but two friction cases - original friction coefficients and with reduced friction, $25 \%$ of original values.
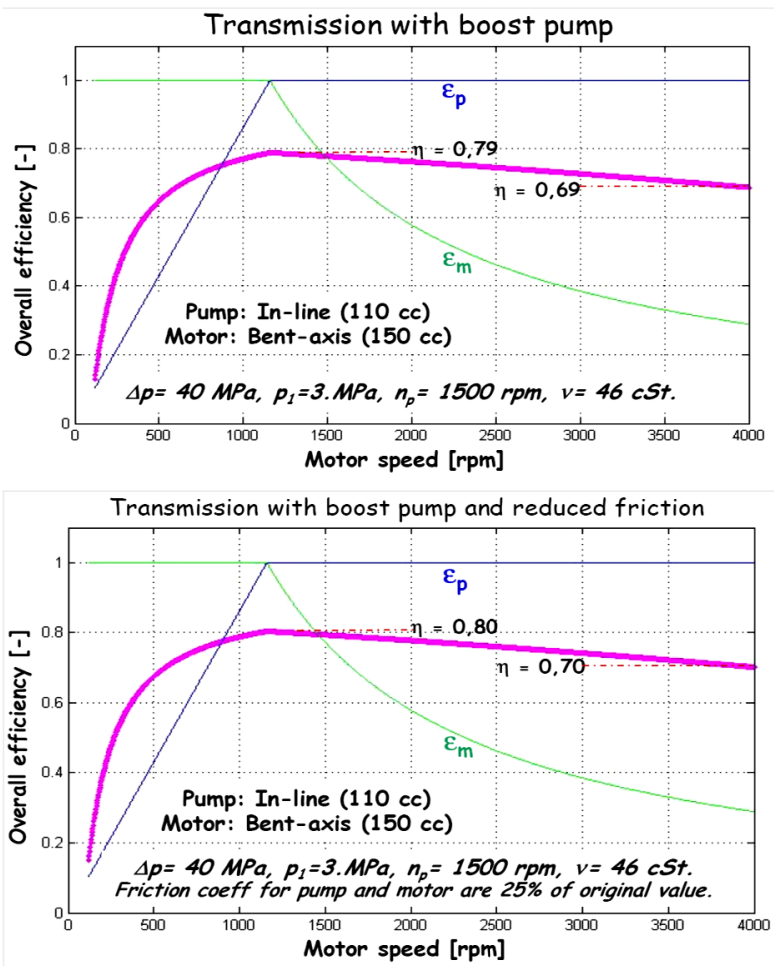

Fig. 11: Simulated transmission overall efficiency versus motor speed, with boost pump, without and with reduced friction.

The figure above shows that this dramatic reduction of friction only will increase the efficiency about $1 \%$. This example demonstrates that, to reach an efficiency gain around 20\%, according to reference [4] and [7], it is not enough to use fluids with friction modifiers, VG- and VI-values as well as shear stability must be adjusted for the application.

It has to be noted that the conducted work presented above is just a theoretical analyses based on semi-empirical efficiency models validated for axial piston units, [1]. 


\subsection{Experimental validation of efficiencies}

In a transmission test-stand at FluMeS lab in Linköping, some efficiency measurements have been conducted. The test object is similar to the transmission shown in Fig. 7. The tests have been performed at max displacement setting of pump and motor $\left(\varepsilon_{\mathrm{p}}=\varepsilon_{\mathrm{m}}=1,0\right)$. Test results are presented in Fig. 12-14.

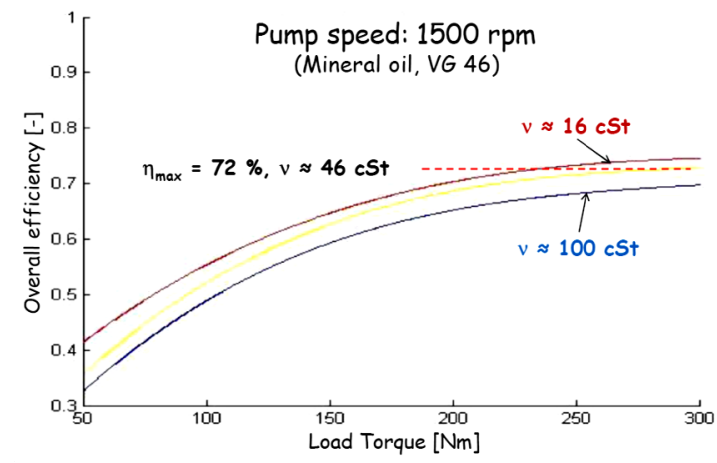

Fig. 12: Measure transmission overall efficiency versus Load Torque, (boost pump incl.), mineral oil VG 46.

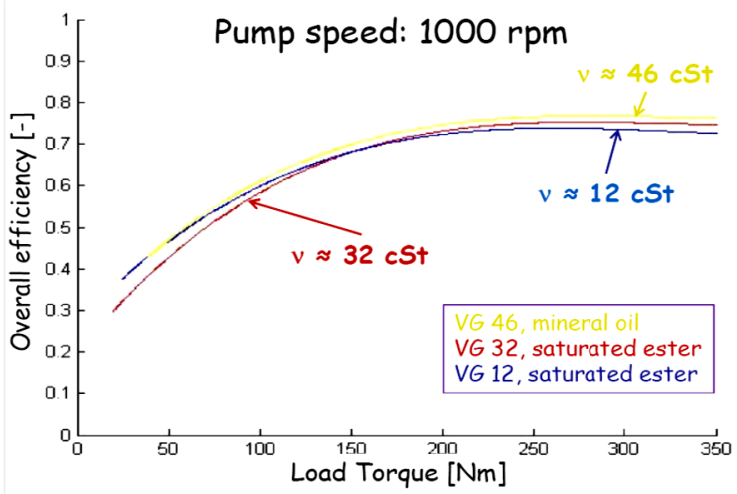

Fig. 13: Measure transmission overall efficiency versus Load Torque, (boost pump incl.), for three different fluids.

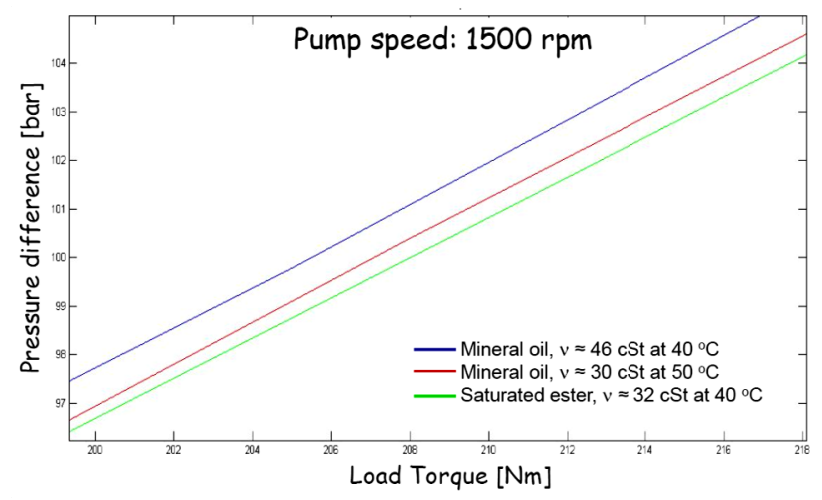

Fig. 14: Measured transmission pressure difference versus Load Torque, (boost pump incl.).

Fig. 12 illustrates the transmission efficiency for mineral oil, at three different fluid temperatures and thereby three different viscosities, 16, 46 and $100 \mathrm{cSt}$. From the lowest to the highest viscosity the change in efficiency is about $5 \%$. It can be noted that this viscosity impact on efficiency corresponds quite well to simulation results presented in this paper (see Fig. 9 and 10).

In Fig. 13 two saturated esters (VG 12 and VG 32) are compared with a mineral oil (VG 46) at low pump speed (1000 rpm). The figure shows that optimal fluid viscosity is load dependent. Increased transmission pressure requires higher viscosity to maximize the efficiency. Also the lubrication property of saturated esters is better than for mineral oil. Fig. 14 demonstrates that the ester cerates lower friction losses than mineral oil.

\section{How to select the best fluid?}

The hydraulic fluid is one on the most important design factors in a hydraulic system and should be considered as a machine element, possible to develop as well as the other hydraulic components in a system.

Fluid selection must be based on a detailed system specification - containing type of components, system pressure range, pump and motor speed range and operation temperature range. Also, expected operation time and reliability must be included in the specification.

Optimal VG-value for the specified system can be found by using theoretical models, which are validated by practical tests. The estimated temperature range for maximum power operation gives the requirement on viscosity index (VI).

Especially in high pressure systems the fluid lubricity is of high importance. It is a well known fact that synthetic esters, gives lower friction and wear than mineral oil. Ester fluids also have higher heat capacity than mineral oils, which results in lower temperature variations. Field tests conducted by the Forestry Research Institute of Sweden, confirms that the steady state fluid temperature in a forest machine, will be reduced $5-10{ }^{\circ} \mathrm{C}$ with use of synthetic esters instead of mineral oils.

Several decades of documented experience shows that the lifetime of a hydraulic system (including the fluid) depends mainly on the cleanliness of the fluid, stability and working temperature. A clean fluid (low amount of particles, low water and air content) with good stability regarding viscosity and acid number that can work in a 
suitable temperature gives a very long system lifetime. It is also a very well known fact that there is a clear connection between the cleanliness, stability and temperature of the fluid. The reason is that particles and water, in combination with high temperature give an accelerating oxidation of the fluid and a faster brake down of the additives.

\section{Conclusions}

The theoretical and practical results presented in this paper, demonstrates that the efficiency of a system is strongly dependent on the viscosity of the hydraulic fluid. The "optimal" viscosity is however, dependent on the pressure level and speed. In an application the viscosity should be selected so that the efficiency is maximized within the pressure and speed where the biggest amount of energy is transferred so that one can reduce the energy losses.

The viscosity index is highly important in mobile systems. It is also stated that synthetic ester fluids gives lower friction losses than standard mineral oil. The shear stability of saturated esters is extremely good compared to mineral oil and unsaturated esters.

However, these facts just represent a part of the information needed to find the "optimal" fluid for a specific application. Besides theoretical facts a solid experience is needed for a satisfactory fluid selection. Finally, this paper demonstrate, that there is no simple answer of the question "How to select the best fluid?". To find a relevant answer, a good choice is to start with a detailed system specification and try to convert the specification into fluid requirements.

\section{Acknowledgement}

This work has been supported by the Swedish Fluid Power Association (IFS).

\section{References}

[1] Rydberg K-E.: On performance optimization and digital control of hydrostatic drives for vehicle applications. Ph.D. thesis no 99, Linköping University, Sweden, 1983.

[2] K Eriksson: Environmental adapted pressure media versus mineral oils, (in Swedish). Presentation at National Hydraulic Days, Linköping, May 30-31, 2007.
[3] High Efficiency Excavator. Info_CCEFP_Excavator. Center for Compact and Efficient Fluid Power, US, March 16, 2011.

[4] A Presher: New Hydraulic Fluids Aimed at Energy Savings. Design News, October 2009.

[5] C K Dustman, Rohmax USA. Performance Benefits of High Viscosity Index Hydraulic Fluids. Lubricants-Russia 2006, Moscow, November 24, 2006.

[6] S Javalagi, S Singireddy: Hydraulic fluid properties and its influence on system performance. Master's Thesis, LIU-IEI-TEK-A--12/01284_SE, Linkoping Univ., 2012.

[7] S Herzog: Improving Fuel Economy and Productivity of Mobile Equipment through Hydraulic Fluid Selection. Evonik Rohmax USA, 2011.

[8] TechBulletin: HYDREX $X_{T M}$ Energy Efficiencies in Mobile Equipment. Petro-Canada, 2010. 